\title{
PRODUÇÃO DE BIOETANOL DE PAPEL DE ESCRITÓRIO DESCARTADO POR Spathaspora passalidarum HMD 14.2 UTILIZANDO HIDRÓLISE ÁCIDA E ENZIMÁTICA
}

\author{
B. R. A. ALENCAR, J. M. T. S. ROCHA, H. G. MOTA e E. R. GOUVEIA. \\ Universidade Federal de Pernambuco, Departamento de Antibióticos
}

RESUMO - O descarte de papel representa cerca de $40 \%$ do total de dejetos sólidos. Visto que a sua composição contém $50 \%$ de celulose e $15 \%$ de hemicelulose, o papel tem potencial para a produção de bioetanol economicamente competitivo. O objetivo deste trabalho foi a produção de bioetanol de papel de escritório descartado utilizando Spathaspora passalidarum HMD 14.2, após hidrólise enzimática. Foram realizados pré-tratamentos em mesa incubadora rotativa e em autoclave, com $8 \% \mathrm{~m} / \mathrm{V}$ de papel e ácido sulfúrico diluído. O pré-tratamento em autoclave, com ácido sulfúrico, gerou a perda de açúcares fermentescíveis, sendo selecionado o prétratamento em mesa incubadora rotativa para a posterior hidrólise enzimática. $\mathrm{O}$ hidrolisado enzimático foi utilizado para a fermentação. Foi realizada a quantificação de glicose, xilose e etanol por cromatografia líquida de alta eficiência. Rendimento de $0,37 \mathrm{~g} / \mathrm{g}$ e eficiência de 72,6 $\%$, em relação à glicose e xilose consumidas pela Spathaspora passalidarum HMD 14.2, foram obtidos, indicando o potencial desta levedura para a produção de bioetanol, a partir de meios contendo glicose e xilose.

\section{INTRODUÇÃO}

A utilização dos açúcares oriundos da etapa de pré-tramento das frações celulósica e hemicelulósica presentes na biomassa de composição lignocelulósica é essencial para a produção viável de etanol. Entretanto, a levedura industrialmente utilizada, Saccharomyces cerevisiae, não pode metabolizar xilose, o segundo açúcar mais abundante em hidrolisados lignocelulósicos (Hahn-Hägerdal et al., 2001). Recentemente, uma levedura fermentadora de xilose, Spathaspora passalidarum, foi isolada de besouro, que preferencialmente habita em madeiras (Nguyen et al., 2006). Estudos com esta levedura mostraram que a mesma pode 
fermentar simultaneamente glucose e xilose, com alto rendimento em etanol (Long et al., 2012).

Os processos de hidrólise enzimática da celulose e da hemicelulose, para obtenção de açucares, em meio aquoso, catalisado por celulases, possuem valores de rendimentos baixos, principalmente devido a elevada cristalinidade da estrutura da celulose. Para tornar a hidrólise mais eficiente, são necessárias etapas de pré-tratamento da matéria-prima, visando aumentar a acessibilidade do substrato à ação enzimática (Mosier et al., 2005).

O objetivo do presente trabalho visa à produção de Bioetanol a partir da fermentação de hidrolisado enzimático de papel de escritório, por Spathaspora passalidarum HMD 14.2.

\section{MATERIAIS E MÉTODOS}

\subsection{Material Lignocelulósico}

Inicialmente, uma quantidade suficiente de papel de escritório descartado foi aproveitada para a execução do presente trabalho. Os equipamentos envolvidos na etapa de pré-tratamento foram uma mesa incubadora rotativa e uma autoclave. Os parâmetros avaliados para os estudos envolvendo a determinação das condições ideais de pré-tratamento e liberação de açucares estão descritos na Tabela 1. Em ambas as estratégias de pré-tratamento, os seguintes procedimentos foram adotados: $8 \% \mathrm{~m} / \mathrm{V}$ de papel, cortados em dimensões de $2 \mathrm{~mm}$, com o auxílio de um perfurador de papel. O material pré-tratado foi, na sequência, centrifugado por 10 min a 10.000 rpm e o material decantado foi encaminhado para a hidrólise enzimática.

\subsection{Hidrólise Enzimática}

As hidrólises foram realizadas em frascos de Erlenmeyers, com tampão citrato de sódio (pH igual a 4,8). Preparações comerciais de celulases (Celluclast $1.5 \mathrm{~L}-2 \mathrm{~mL} ; 119 \mathrm{FPU} / \mathrm{mL}$ ) e $\beta$-glucosidase $(1 \mathrm{~mL})$, ambas da Novozyme, foram utilizadas. A atividade enzimática da Celluclast 1.5 L foi determinada com unidade de papel de filtro por $\mathrm{mL}$, segundo o método de Ghose (1987). Os frascos, em todas as hidrólises enzimáticas, foram mantidos em mesa incubadora rotativa, a $50^{\circ} \mathrm{C}$ e $150 \mathrm{rpm}$. Após a filtração das amostras em membrana de $0,45 \mu \mathrm{m}$, os filtrados foram utilizados para a quantificação de glicose e xilose por cromatografia líquida 
de alta eficiência.

Tabela 1. Condições dos pré-tratamentos em mesa incubadora rotativa e em autoclave.

\begin{tabular}{c|c|c|c|c}
\hline Pré-tratamento & $\begin{array}{r}\text { Temperatura } \\
\left({ }^{\circ} \mathrm{C}\right)\end{array}$ & $\begin{array}{r}\text { Agitação } \\
(\mathrm{rpm})\end{array}$ & $\begin{array}{r}\mathrm{H}_{2} \mathrm{SO}_{4} \\
(\% \mathrm{~V} / \mathrm{V})\end{array}$ & $\begin{array}{c}\text { Tempo } \\
(\mathrm{h})\end{array}$ \\
\hline Mesa incubadora rotativa & 50 & 50 & 1 & 3 \\
Autoclave & 121 & - & 5 & 1 \\
\hline
\end{tabular}

\subsection{Microrganismo e Meios de Cultura}

Foi utilizada uma linhagem de levedura, Spathaspora passalidarum HMD 14.2, cedida pelo Departamento de Micologia do Instituto de Ciências Biológicas da UFMG. O meio de cultura utilizado para a conservação, em tubo de ensaio inclinado, foi composto de glicose (20 g/L), extrato de levedura (4 g/L), peptona (3 g/L) e ágar (15 g/L). Na preparação do inoculo, também foi utilizada a mesma composição de meio, mas sem adição de ágar. $\mathrm{O} \mathrm{pH}$ foi 6,8 $\pm 0,2 \mathrm{em}$ ambos os meios. As fermentações foram realizadas utilizando um meio de cultura contendo $\left(\mathrm{NH}_{4}\right)_{2} \mathrm{SO}_{4}(2 \mathrm{~g} / \mathrm{L}), \mathrm{KH}_{2} \mathrm{PO}_{4}(2 \mathrm{~g} / \mathrm{L}), \mathrm{MgSO}_{4} \cdot 7 \mathrm{H}_{2} \mathrm{O}$ $(0,75 \mathrm{~g} / \mathrm{L})$ e extrato de levedura $(4 \mathrm{~g} / \mathrm{L})$. Estes nutrientes foram dissolvidos no hidrolisado enzimático. A fonte de glicose foi derivada da celulose (glicose) e hemicelulose (xilose) presentes no hidrolisado enzimático do papel.

\subsection{Fermentações do Hidrolisado Enzimático}

Na preparação do inóculo, a linhagem foi repicada em tubos de ensaio contendo o meio de conservação, os quais foram mantidos em estufa a $30^{\circ} \mathrm{C}$ durante 24 horas. Após esse período, todo o microrganismo do tubo de ensaio foi transferido para frascos de 
Erlenmeyer de $250 \mathrm{~mL}$ contendo $50 \mathrm{~mL}$ do meio de inoculo. O frasco foi submetido a 200 rpm e $30^{\circ} \mathrm{C}$, por 7 horas, em mesa incubadora rotativa. Posteriormente, todo o volume de suspensão do frasco de Erlenmeyer, foi filtrado em membrana de 0,45 $\square \mathrm{m}$. Um volume de $10 \mathrm{~mL}$ de água destilada estéril foi adicionado à biomassa e, em seguida, $5 \mathrm{~mL}$ da suspensão microbiana foi transferida para um dos dois frascos de Erlenmeyer de $125 \mathrm{~mL}$ contendo $45 \mathrm{~mL}$ do meio de fermentação. Os frascos foram mantidos a $30{ }^{\circ} \mathrm{C}$ e sem agitação, durante 24 horas.

\subsection{Rendimento}

O rendimento das hidrólises com relação à formação de glicose e xilose foi determinado pela equação (1).

$$
Y(\%) \square \frac{\mathrm{G} \square \mathrm{X}}{\mathrm{m}} * V * 100
$$

G: concentração de glicose (em g/L);

$\mathrm{X}$ : concentração de xilose (em g/L);

$\mathrm{V}$ : volume da mistura reacional (em L);

m: massa de papel (em g).

$\mathrm{O}$ rendimento em relação à formação de etanol foi determinado de acordo com a equação (2).

$$
Y_{P / S} \square \frac{\lambda E}{\lambda S}
$$

$\leftrightarrows \mathrm{E}:$ variação da concentração de etanol (em g/L);

$\leftrightarrows \mathrm{S}$ : variação da concentração de glicose e xilose (em g/L).

A eficiência da fermentação foi determinada pela equação (3), considerando o rendimento teórico igual a $0,511 \mathrm{~g} / \mathrm{g}$.

$$
E_{f} \square \frac{Y_{P / S}}{0,511} * 100
$$


A produtividade volumétrica foi determinada pela equação (4).

$$
Q_{P} \square \frac{E}{t}
$$

E: concentração de etanol com 24 horas de fermentação (em g/L);

t: tempo final da fermentação (24 horas).

\subsection{Métodos Analíticos}

Foram retiradas alíquotas, ao final de cada hidrólise e das fermentações. As amostras foram centrifugadas a $10.000 \mathrm{rpm}$ durante $10 \mathrm{~min}$, sendo o sobrenadante destinado para quantificação de carboidratos e etanol, após de filtração em membrana de celulose, com uma porosidade de 0,45 \m. A quantificação das amostras, contendo glicose, xilose e etanol, foi realizada por cromatografia líquida de alta eficiência (CLAE) em cromatógrafo da Agilent HP 1100, com uma coluna de troca catiônica HPX-87H+ (300 mm x 7,8 mm, Bio-Rad Laboratories, Richmond, CA, USA), a $60^{\circ} \mathrm{C}$ e detecção por índice de refração. Foram realizadas injeções de $5 \mu \mathrm{L}$, com vazão de $0,6 \mathrm{~mL} / \mathrm{min}$, utilizando como fase móvel $\mathrm{H}_{2} \mathrm{SO}_{4}$ a $5 \mathrm{mM}$.

\section{RESULTADOS}

O pré-tratamento com ácido sulfúrico diluído em mesa incubadora rotativa foi fundamental para as hidrólises enzimáticas com alta carga de sólidos (8 ou $10 \% \mathrm{~m} / \mathrm{V})$. Na produção de bioetanol, a concentração inicial de açúcares fermentescíveis é um fator preponderante para a obtenção de maior concentração do produto. Daí a seleção de $8 \% \mathrm{~m} / \mathrm{V}$ para a carga de enzimas utilizada neste trabalho, uma vez que nesta condição foi obtida a maior concentração de glicose e xilose.

Foi realizado um pré-tratamento apenas com $8 \% \mathrm{~m} / \mathrm{V}$ em autoclave, uma vez que este foi o melhor resultado obtido. As condições operacionais foram descritas na Tabela 1. Comparandose a fração líquida, após os pré-tratamentos, aquela resultante do uso da autoclave (B) apresentou 
concentrações de glicose e de xilose iguais a 9,09 g/L e 9,10 g/L, respectivamente (Figura 1). Por outro lado, o pré-tratamento realizado em mesa incubadora rotativa (A) não apresentou quantidades significativas de glicose e xilose na fração líquida, mantendo a concentração de açucares na fração sólida e disponível para a ação enzimática.

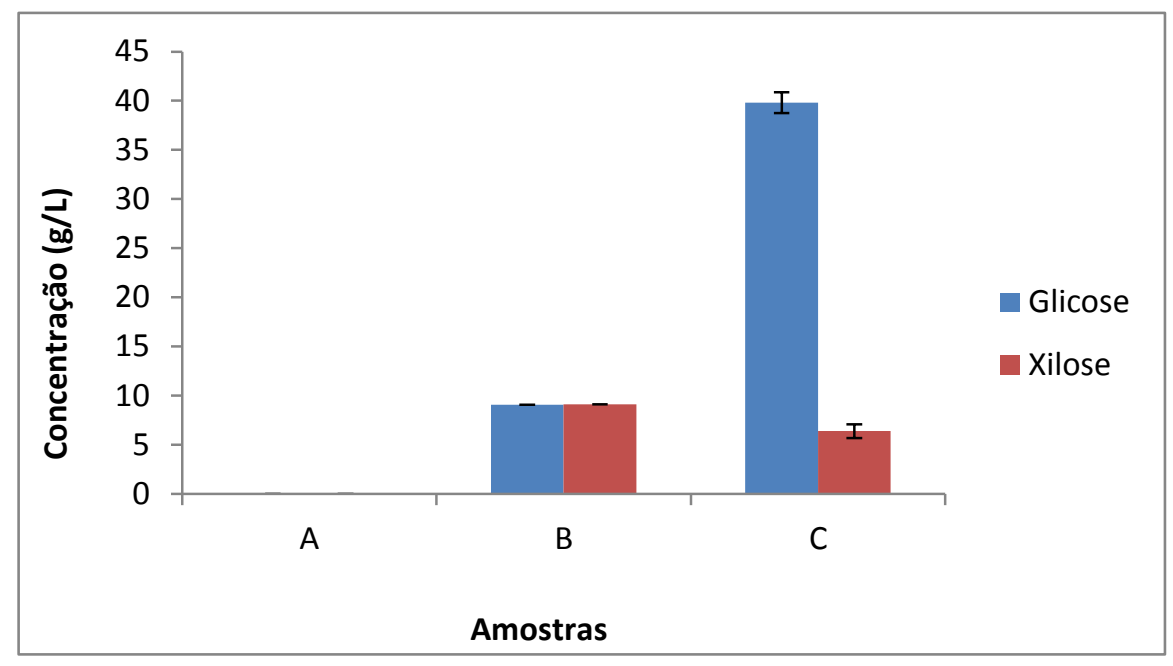

Figura 1. Concentração de glicose e xilose na fração líquida após pré-tratamento com (A) ácido sulfúrico $(1 \% \mathrm{v} / \mathrm{v})$ em mesa incubadora rotativa a $50^{\circ} \mathrm{C}, 150 \mathrm{rpm}$, por 3 horas; (B) com ácido sulfúrico $(5 \% \mathrm{~V} / \mathrm{V})$ na autoclave a $121{ }^{\circ} \mathrm{C}$, por 1 hora e $(\mathrm{C})$ após hidrólise enzimática.

A Figura 1 (C) apresenta as concentrações de glicose e de xilose após a hidrólise enzimática da fração sólida obtida após o pré-tratamento em mesa incubadora rotativa. Após 96 horas de hidrólise enzimática desta fração sólida, foram obtidas concentrações de glicose e xilose iguais a $40 \mathrm{~g} / \mathrm{L}$ e $6 \mathrm{~g} / \mathrm{L}$, respectivamente. O hidrolisado enzimático (A) foi utilizado para a fermentação. As Tabelas 2 e 3 apresentam as concentrações de glicose, xilose e etanol obtidas com 24 horas de fermentação, bem como os parâmetros da fermentação (rendimento, produtividade e eficiência).

Tabela 2. Concentrações de glicose, xilose e etanol no início e no final da fermentação do hidrolisado enzimático com $8 \% \mathrm{~m} / \mathrm{V}$ e pré-tratamento em mesa incubadora rotativa.

\begin{tabular}{l|l|l} 
Composto & Concentração inicial & Concentração final \\
\hline
\end{tabular}




\begin{tabular}{c|c|c}
\hline & $(\mathrm{g} / \mathrm{L})$ & $(\mathrm{g} / \mathrm{L})$ \\
\hline Glicose & 32,39 & 0,60 \\
Xilose & 6,03 & 1,52 \\
Etanol & 0,00 & 13,69 \\
\hline
\end{tabular}

A glicose foi praticamente consumida durante a fermentação. Por outro lado, após 24 horas, a concentração de xilose foi cerca de $2 \mathrm{~g} / \mathrm{L}$. Isso indica a preferência de consumo da glicose pela S. passalidarum HMD 14.2. Segundo Long et al. (2012), S. passalidarum assimila simultaneamente glicose, celobiose e xilose durante a produção de etanol. Lima et al. (2014), em fermentação de hidrolisado ácido de papel de escritório descartado, por $S$. passalidarum HMD 14.2 também observaram um consumo preferencial da glicose.

Tabela 3. Produtividade, rendimento e eficiência obtida na fermentação do hidrolisado enzimático com $8 \% \mathrm{~m} / \mathrm{V}$ e pré-tratamento em mesa incubadora rotativa.

\begin{tabular}{c|c}
\hline Parâmetro & Valor \\
\hline $\mathrm{Q}_{\mathrm{P}}(\mathrm{g} / \mathrm{L} . \mathrm{h})$ & 0,57 \\
$\mathrm{Y}_{\mathrm{P} / \mathrm{S}}(\mathrm{g} / \mathrm{g})$ & 0,37 \\
Eficiência (\%) & 72,65 \\
\hline
\end{tabular}

Rendimento de $0,37 \mathrm{~g} / \mathrm{g}$ e eficiência de 72,6 \%, em relação à glicose e xilose, consumidas pela Spathaspora passalidarum HMD 14.2 foram obtidos, indicando o potencial desta levedura para a produção de bioetanol a partir de meios contendo glicose e xilose.

Wanderley et al. (2013) obtiveram 0,39 g/g e 78,5 \% de eficiência, em fermentações de hidrolisados enzimáticos de bagaço de cana-de-açúcar após o uso de pré-tratamento pela técnica da explosão a vapor e deslignificação alcalina, utilizando Saccharomyces cerevisiae UFPEDA 1238. 


\section{REFERÊNCIAS}

HAHN-HAGERDAL, B.; WAHLBOM, C. F.; GARDONYI, M.; VAN ZYL, W. H.; CORDERO, R.; JONSSON, L. J. Metabolic engineering of Saccharomyces cerevisiae for xylose utilisation. Advance in Biochemical Engineering and Biotechnology, v. 73, p. 53-84, 2001.

LIMA, D. A.; LUNA, R. L. N.; MARTÍN, C.; GOUVEIA, E. R. Comparison of bioethanol production from acid hydrolyzates of waste office paper using Saccharomyces cerevisiae and Spathaspora passalidarum. Cellulose Chemistry and Technology, 2014 (aceito).

LONG, T. M.; SU, YI-KAL; HEADMAN, J.; HIGBEE, A.; WILLIS, L. B.; JEFFRIES, T. W. Cofermentation of Glucose, Xylose, and Cellobiose by the Beetle- Associated Yeast Spathaspora passalidarum. Current Opinion in Chemical Biology, v. 78, p. 5492-5500, 2012.

MOSIER, N. et al. Features of promising technologies for pretreatment of lignocellulosic biomass. Bioresource Technology, v. 96, p. 673-686, 2005.

NGUYEN, H.; SUH, S. O.; MARSHALL, C. J.; BLACKWELL, M. Morphological and ecological similarities: wood-boring beetles associated with novel xylose-fermenting yeasts, Spathaspora passalidarum sp. nov. and Candida jeffriessii sp. nov. Mycology Research, v. 110, p. 1232 -1241, 2006.

WANDERLEY, M. C. A.; MARTÍN, M.; ROCHA, G. L. M.; GOUVEIA, E. R. Increase in ethanol production from sugarcane bagasse based on combined pretreatments and fed-batch enzymatic hydrolysis. Bioresource Technology, v. 128, p. 448-453, 2013. 